\title{
Finite-Time Passivity and Passification Design for Markovian Jumping Systems with Mode-Dependent Time-Varying Delays
}

\author{
Chao Ma \\ School of Automation and Electrical Engineering, University of Science and Technology Beijing, Beijing 100083, China \\ Correspondence should be addressed to Chao Ma; cma@ustb.edu.cn
}

Received 29 September 2016; Revised 8 February 2017; Accepted 16 February 2017; Published 7 March 2017

Academic Editor: Chuanxi Qian

Copyright ( 2017 Chao Ma. This is an open access article distributed under the Creative Commons Attribution License, which permits unrestricted use, distribution, and reproduction in any medium, provided the original work is properly cited.

\begin{abstract}
This paper investigates the finite-time passivity and passification design problem for a class of Markovian jumping systems with mode-dependent time-varying delays. By employing the Lyapunov-Krasovskii functional method, delay-dependent sufficient criteria are derived to ensure the mean-square stochastically finite-time passivity. Based on the established results, mode-dependent passification controller is further designed in terms of linear matrix inequalities, such that the prescribed passive performance index of the resulting closed-loop system can be satisfied. Finally, two illustrative examples are given to show the effectiveness of the obtained theoretical results.
\end{abstract}

\section{Introduction}

During the past decade, Markovian jumping systems have received much attention since they can provide a unified framework in modeling practical systems in the real world including networked control systems, chaos systems, power systems, and biological systems (e.g., [1-3] and the references therein). It should be pointed out that time delays have inevitably emerged in Markovian jumping systems, which would affect the dynamical characteristics and system performances. In particular, substantial studies have found that time delays in Markovian jumping systems often vary according to different system modes. As a consequence, a large number of effective approaches for analysis and synthesis of Markovian jumping systems with mode-dependent timevarying delays have been reported in the literature [4-7].

On another research front line, as a particular form of dissipativity, passivity theory has played a significant role in dealing with many types of dynamical systems by relating the system inputs and outputs, especially for complex systems $[8,9]$. Currently, many efforts have been devoted to the researches on control systems in the passivity framework due to its features, which have achieved satisfying results among different performance indices such as $H_{\infty}$ index and $L_{2}-L_{\infty}$ index. As a result, controllers can be designed to guarantee the passivity of the resulting closed-loop system, which gives rise to the so-called passification problems [1012]. It is noted that the relation between stability and passivity can also be revealed from the energy point of view, as well as the controller design problems. While considering Markovian jumping systems, mode-dependent controllers are often designed to reduce the conservatism due to practical conditions. Encouragingly, some burgeoning results in this aspect have been available so far [13-16]. Nevertheless, it should be pointed out that the above results were all established in the context of an infinite-time interval.

Recently, finite-time control issues of dynamical systems have been extensively studied due to their practical backgrounds. Different from the asymptotic stability in the merely infinite-time interval, the finite-time stability theory can ensure the asymptotic behavior over a finite-time interval (often a short interval), which means that the system states should be below the prescribed upper bound during the given time-intervals. To mention a few, [17] addressed the finite-time $H_{\infty}$ control problem of uncertain switched linear neutral systems with time-varying delays. Reference [18] concerned the finite-time stochastic synchronization of genetic regulatory networks. Reference [19] investigated the finite-time sampled-data control for switching T-S fuzzy systems. Reference [20] solved the finite-time filtering for switched linear systems with a mode-dependent average dwell time. These results show that, by utilizing the finite-time 
techniques, the transient performance of the dynamical systems can be well analyzed and is more practical for actual conditions. However, to the best of the authors' knowledge, the finite-time passivity and passification design problems for Markovian jumping systems with mode-dependent timevarying delays still have not been fully explored and remain challenging, let alone those under the finite-time theory framework.

Motivated by the aforementioned observations, in this paper, we aim at solving the finite-time control problem of Markovian jumping systems with mode-dependent timevarying delays and disturbances by a passivity-based scheme. Compared with the previous results, the main contributions of this paper can be summarized in two points: (1) this paper makes one of the first steps to deal with the passivity and passification for Markovian jumping systems with mode-dependent time-varying delays; (2) the corresponding mode-dependent controller is designed in the finite-time framework, which is more applicable and practical in the applications. By applying the finite-time theory and constructing appropriate mode-dependent Lyapunov-Krasovskii functionals, the resulting closed-loop system can be stochastically finite-time bounded with the prescribed passivity performance level. Furthermore, the mode-dependent controller is designed by using the matrix transformation approach. The rest of this paper is organized as follows. In Section 2, the Markovian jumping system model is introduced and some essential lemmas and definitions are provided for the formulated problem. Our main results on the finite-time passivity and passification design are given in Section 3. Section 4 presents two numerical examples to demonstrate the effectiveness of our proposed control method. The paper is concluded in Section 5.

Notation. The notations throughout this paper are quite standard. $\mathbb{R}^{n}$ denotes $n$ dimensional Euclidean space, and $\mathbb{R}^{m \times n}$ represent the set of all $m \times n$ real matrices. The notation $P>0$ means $P$ is real symmetric and positive definite, and the superscript " $T$ " denotes matrix transposition. $\lambda_{\text {min }}(A)$ and $\lambda_{\text {max }}(A)$ denote the minimum and the maximum eigenvalues of the corresponding matrix $A$, respectively. $\operatorname{sym}\{A\}$ denotes $A+A^{T} \cdot \mathscr{L}_{2}[0, \infty)$ denotes the space of square-integrable vector functions over $[0, \infty) .(\Omega, \mathscr{F}, \mathscr{P})$ is a probability space, $\Omega$ is the sample space, $\mathscr{F}$ is the $\sigma$-algebra of subsets of the sample space, and $\mathscr{P}$ is the probability measure on $\mathscr{F}$. $\mathbb{E}\{\cdot\}$ denotes the mathematics expectation of the stochastic process or vector. Moreover, in symmetric block matrices, * is used as an ellipsis for the terms that are introduced by symmetry and $\operatorname{diag}\{\cdots\}$ denotes a block-diagonal matrix. In the sequel, if not explicitly states, all matrices are assumed to have compatible dimensions.

\section{Problem Formulation and Preliminaries}

Fix a probability space $(\Omega, \mathscr{F}, \mathscr{P})$ and consider the following class of Markovian jumping systems with mode-dependent time-varying delays:

$$
\dot{x}(t)=A(r(t)) x(t)+A_{d}(r(t)) x\left(t-d_{r(t)}(t)\right)
$$

$$
\begin{aligned}
& +B(r(t)) u(t)+B_{\omega}(r(t)) \omega(t), \\
& y(t)=C(r(t)) x(t)+C_{d}(r(t)) x\left(t-d_{r(t)}(t)\right) \\
& +D_{\omega}(r(t)) \omega(t), \\
& \quad x(t)=\phi(t), \quad r(t)=r_{0}, \forall t \in[-\bar{d}, 0],
\end{aligned}
$$

where $x(t) \in \mathbb{R}^{n}$ denotes the state vector of the Markovian jumping systems, $u(t) \in \mathbb{R}^{n}$ represents the control input, $\omega(t) \in \mathbb{R}^{m}$ is the unknown disturbance input which belongs to $\mathscr{L}_{2}[0, \infty)$ and satisfies the condition $\int_{0}^{T} \omega^{T}(t) \omega(t) \leq$ $\delta, \delta>0, y(t) \in \mathbb{R}^{s}$ denotes the measurement output, $d_{r(t)}(t)$ is the mode-dependent time-varying delay satisfying $0 \leq d_{r(t)}(t) \leq \bar{d}$, and $\dot{d}_{r(t)}(t) \leq \mu<1$, where $\bar{d}$ and $\mu$ are known positive constants.

$r(t)(t \geq 0)$ denotes a right-continuous Markov process on $(\Omega, \mathscr{F}, \mathscr{P})$ taking values in a finite set $\mathscr{I}=\{1, \ldots, N\}$, where the generator $\Theta=\left\{\pi_{i j}\right\}, \forall i, j \in \mathscr{I}$, is described by

$$
\begin{aligned}
\operatorname{Pr}(r(t+\Delta t)=j: r(t)=i) \\
\quad= \begin{cases}\pi_{i j} \Delta t+o(\Delta t), & \text { if } i \neq j, \\
1+\pi_{i i} \Delta t+o(\Delta t), & \text { if } i=j\end{cases}
\end{aligned}
$$

with $\Delta t>0$ and $\lim (o(\Delta t) / \Delta t)=0$ and $\pi_{i j} \geq 0(i, j \in \mathscr{I}, j \neq$ $i$ ) is the transition rate from mode $i$ at time $t$ to mode $j$ at time $t+\Delta t$, while $\pi_{i i}=-\sum_{j=1, j \neq i}^{N} \pi_{i j} \forall i \in \mathscr{I}$.

Moreover, all $A(r(t)), A_{d}(r(t)), B(r(t)), B_{\omega}(r(t)), C(r(t))$, $C_{d}(r(t))$, and $D_{\omega}(r(t))$ are known constant matrices with appropriate dimensions for each $r(t) \in \mathscr{I}$.

In this paper, the following mode-dependent statefeedback controller is designed:

$$
u(t)=K(r(t)) x(t)
$$

where $K(r(t))$ is the controller gain matrix with appropriate dimensions to be determined later.

Then, the closed-loop system can be obtained by combining (1) and (3) that

$$
\begin{aligned}
\dot{x}(t)= & {[A(r(t))+B(r(t)) K(r(t))] x(t) } \\
& +A_{d}(r(t)) x\left(t-d_{r(t)}(t)\right) \\
& +B_{\omega}(r(t)) \omega(t), \\
y(t)= & C(r(t)) x(t)+C_{d}(r(t)) x\left(t-d_{r(t)}(t)\right) \\
& +D_{\omega}(r(t)) \omega(t), \\
& x(t)=\phi(t), r(t)=r_{0}, \quad \forall t \in[-\bar{d}, 0] .
\end{aligned}
$$


For notational simplicity, each possible $r(t)$ is denoted by the index $i, i \in \mathscr{I}$, such that matrix $A(r(t))$ is denoted by $A_{i}$ and so on. Consequently, (4) can be rewritten as follows:

$$
\begin{array}{r}
\dot{x}(t)=\left(A_{i}+B_{i} K_{i}\right) x(t)+A_{d i} x\left(t-d_{i}(t)\right)+B_{\omega i} \omega(t), \\
y(t)=C_{i} x(t)+C_{d i} x\left(t-d_{i}(t)\right)+D_{\omega i} \omega(t), \\
x(t)=\phi(t), r(t)=r_{0}, \forall t \in[-\bar{d}, 0] .
\end{array}
$$

Before proceeding, some essential definitions are provided as follows.

Definition 1 (MSSFTS). For some given constants $c_{1}>0, \delta>$ 0 , and $T>0$ and symmetric matrix $W>0$, system (5) with $\omega(t) \equiv 0$ is said to be mean-square stochastically finite-time stable (MSSFTS) with respect to $\left(c_{1}, c_{2}, T, W\right)$, if there exist constants $c_{2}>c_{1}$, such that

$$
\begin{aligned}
\mathbb{E}\left\{x^{T}\left(t_{0}\right) W x\left(t_{0}\right)\right\} \leq c_{1} \Longrightarrow & \mathbb{E}\left\{x^{T}(t) W x(t)\right\} \leq c_{2}, \\
& \forall t_{0} \in[-\bar{d}, 0], t \in[0, T]
\end{aligned}
$$

Definition 2 (MSSFTB). For some given constants $c_{1}>0$, $\delta>0$, and $T>0$ and symmetric matrix $W>0$, system (5) is said to be mean-square stochastically finite-time bounded (MSSFTB) with respect to $\left(c_{1}, c_{2}, \delta, T, W\right)$, if there exist constants $c_{2}>c_{1}$, such that

$$
\begin{aligned}
& \mathbb{E}\left\{x^{T}\left(t_{0}\right) W x\left(t_{0}\right)\right\} \leq c_{1}, \\
& \int_{0}^{T} \omega^{T}(t) \omega(t) d t \leq \delta \\
& \Downarrow \\
& \mathbb{E}\left\{x^{T}(t) W x(t)\right\} \leq c_{2}, \\
& \forall t_{0} \in[-\bar{d}, 0], t \in[0, T] .
\end{aligned}
$$

Definition 3 (MSSFTP). For some given constants $c_{1}>0$, $\delta>0$, and $T>0$ and symmetric matrix $W>0$, system (5) is said to be mean-square stochastically finite-time passive (MSSFTP) with respect to $\left(c_{1}, c_{2}, \delta, T, W\right)$, if there exist constants $c_{2}>c_{1}$, such that system (5) is MSSFTB and there exists a positive constant $\gamma$, such that

$$
2 \mathbb{E}\left\{\int_{0}^{T} y^{T}(t) \omega(t) d t\right\} \geq-\gamma \int_{0}^{T} \omega^{T}(t) \omega(t) d t
$$

Remark 4. The MSSFTP index is introduced to deal with the external disturbances. It is worth mentioning that the concept of being Lyapunov mean-square stochastic stable and being MSSFTS is different, since a MSSFTS system may not be Lyapunov mean-square stochastically stable and vice versa. When there are no external disturbances, the MSSFTB system can be reduced to MSSFTS system. By introducing the MSSFTP performance index $\gamma$, the formulated passification problem can be solved in finite time.
The purpose of this paper is to design a mode-dependent passification controller for system (1) such that the closedloop system (5) is MSSFTP. To this end, the following lemma is introduced for the subsequent analysis.

Lemma 5 (see [21]). For any positive symmetric constant matrix $\mathscr{M} \in \mathbb{R}^{n \times n}$, scalars $h_{1}, h_{2}$ satisfying $h_{1}<h_{2}$, a vector function $\phi:\left[h_{1}, h_{2}\right] \rightarrow \mathbb{R}^{n}$, such that the integrations concerned are well defined, and then

$$
\begin{aligned}
& \left(\int_{h_{1}}^{h_{2}} \phi(s) d s\right)^{T} \mathscr{M}\left(\int_{h_{1}}^{h_{2}} \phi(s) d s\right) \\
& \quad \leq\left(h_{2}-h_{1}\right)\left(\int_{h_{1}}^{h_{2}} \phi^{T}(s) \mathscr{M} \phi(s) d s\right) .
\end{aligned}
$$

\section{Main Results}

In this section, delay-dependent sufficient conditions are first established for the proposed finite-time control problem based on passivity theory. Then, the corresponding controller gain design procedure is provided in terms of LMIs.

Theorem 6. For given positive constants $c_{1}, \delta$ and a symmetric matrix $W>0$, system (5) is MSSFTB with respect to $\left(c_{1}, c_{2}, \delta, T, W\right)$, if there exist a positive constant $c_{2}$ and modedependent symmetric matrices $P_{i}>0$, symmetric matrices $Q>0$ and $R>0$, such that $\Pi_{i}<0$ holds for each mode $i \in \mathscr{I}$ and $c_{1}\left(\lambda_{1}+d \lambda_{2}+d \lambda_{3}\right)+\delta\left(1-e^{-a T}\right)<c_{2} \lambda_{4} e^{-a T}$, where

$$
\begin{aligned}
\Pi_{i} \triangleq & {\left[\begin{array}{cc}
\Pi_{i 1} & \Pi_{i 2} \\
* & \Pi_{i 3}
\end{array}\right], } \\
\Pi_{i 1} \triangleq & \operatorname{sym}\left\{P_{i} A_{i}\right\}+\operatorname{sym}\left\{P_{i} B_{i} K_{i}\right\}+Q+R-\alpha P_{i} \\
& +\sum_{j=1}^{N} \pi_{i j} P_{j}, \\
\Pi_{i 2} \triangleq & {\left[\begin{array}{cccc}
P_{i} A_{d i} & 0 & 0 & P_{i} B_{\omega i} \\
-(1-\mu) Q & 0 & 0 & 0
\end{array}\right], } \\
\Pi_{i 3} \triangleq & \operatorname{diag}\left\{-R, \frac{\bar{\pi}}{\bar{d}} Q,-\alpha I\right\},
\end{aligned}
$$

with $\bar{\pi} \triangleq \max _{i \in \mathscr{I}}\left\{-\pi_{i i}\right\}, \mathscr{P}_{i} \triangleq W^{-1 / 2} P_{i} W^{-1 / 2}, \mathbb{Q} \triangleq$ $W^{-1 / 2} Q W^{-1 / 2}$, and $\mathscr{R} \triangleq W^{-1 / 2} R W^{-1 / 2}$ and $\lambda_{1} \triangleq \lambda_{\max }\left(\mathscr{P}_{i}\right)$, $\lambda_{2} \triangleq \lambda_{\text {max }}(\mathscr{Q}), \lambda_{3} \triangleq \lambda_{\text {max }}(\mathscr{R})$, and $\lambda_{4} \triangleq \lambda_{\text {min }}\left(\mathscr{P}_{i}\right)$.

Proof. Choose the Lyapunov-Krasovskii function candidate as follows:

$$
V(t, i)=\sum_{k=1}^{3} V_{k}(t, i)
$$

where

$$
\begin{aligned}
& V_{1}(t, i) \triangleq x^{T}(t) P_{i} x(t) \\
& V_{2}(t, i) \triangleq \int_{t-d_{i}(t)}^{t} x^{T}(\varphi) Q x(\varphi) d \varphi \\
& V_{3}(t, i) \triangleq \int_{t-\bar{d}}^{t} x^{T}(\varphi) R x(\varphi) d \varphi .
\end{aligned}
$$


Then, define the infinitesimal operator $\mathscr{L}$ of $V(t, i)$ as follows:

$$
\mathscr{L} V(t, i) \triangleq \lim _{\Delta \rightarrow 0^{+}} \frac{1}{\Delta}\{\mathbb{E}\{V(t+\Delta, i) \mid t\}-V(t, i)\} .
$$

For each mode $i \in \mathscr{I}$, the derivative of (11) along the solution of system (5) can be obtained as

$$
\begin{aligned}
& \mathscr{L} V_{1}(t, i)=\dot{x}^{T}(t) P_{i} x(t)+x^{T}(t) P_{i} \dot{x}(t) \\
& +\sum_{j=1}^{N} \pi_{i j} x^{T}(t) P_{j} x(t)=2 x^{T}(t) P_{i}\left[\left(A_{i}+B_{i} K_{i}\right) x(t)\right. \\
& \left.+A_{d i} x\left(t-d_{i}(t)\right)+B_{\omega i} \omega(t)\right] \\
& +\sum_{j=1}^{N} \pi_{i j} x^{T}(t) P_{j} x(t) \\
& \mathscr{L} V_{2}(t, i)=x^{T}(t) Q x(t)-\left(1-\dot{d}_{i}(t)\right) x^{T}\left(t-d_{i}(t)\right) \\
& \text { - } Q x\left(t-d_{i}(t)\right)+\sum_{j=1}^{N} \pi_{i j} \int_{t-d_{j}(t)}^{t} x^{T}(\varphi) Q x(\varphi) d \varphi, \\
& \leq x^{T}(t) Q x(t)-(1-\mu) x^{T}\left(t-d_{i}(t)\right) Q x\left(t-d_{i}(t)\right) \\
& +\sum_{j=1}^{N} \pi_{i j} \int_{t-d_{j}(t)}^{t} x^{T}(\varphi) Q x(\varphi) d \varphi, \\
& \mathscr{L} V_{3}(t, i)=x^{T}(t) R x(t)-x^{T}(t-\bar{d}) R x(t-\bar{d}) . \\
& \text { Since } \pi_{i j} \geq 0, \forall i \neq j \text {, and } \pi_{i i} \leq 0 \text {, one has } \\
& \sum_{j=1}^{N} \pi_{i j} \int_{t-d_{j}(t)}^{t} x^{T}(\varphi) Q x(\varphi) d \varphi \\
& =\sum_{j=1, i \neq j}^{N} \pi_{i j} \int_{t-d_{j}(t)}^{t} x^{T}(\varphi) Q x(\varphi) d \varphi \\
& +\pi_{i i} \int_{t-d_{j}(t)}^{t} x^{T}(\varphi) Q x(\varphi) d \varphi \\
& \leq \sum_{j=1, i \neq j}^{N} \pi_{i j} \int_{t-\bar{d}}^{t} x^{T}(\varphi) Q x(\varphi) d \varphi \\
& =-\pi_{i i} \int_{t-\bar{d}}^{t} x^{T}(\varphi) Q x(\varphi) d \varphi \\
& \leq \bar{\pi} \int_{t-\bar{d}}^{t} x^{T}(\varphi) Q x(\varphi) d \varphi \text {. }
\end{aligned}
$$

Moreover, it follows by Lemma 5 that

$$
\begin{aligned}
& \bar{\pi} \int_{t-\bar{d}}^{t} x^{T}(\varphi) Q x(\varphi) d \varphi \\
& \quad \leq \frac{\bar{\pi}}{\bar{d}} \int_{t-\bar{d}}^{t} x^{T}(\varphi) d \varphi Q \int_{t-\bar{d}}^{t} x(\varphi) d \varphi .
\end{aligned}
$$

The following function is defined:

$$
J \triangleq \mathscr{L} V(t, i)-\left[\alpha V(t, i)+\alpha \omega^{T}(t) \omega(t)\right]
$$

Consequently, it can be deduced that

$$
\begin{aligned}
& J+\alpha \int_{t-d_{i}(t)}^{t} x^{T}(\varphi) Q x(\varphi) d \varphi \\
& \quad+\alpha \int_{t-\bar{d}}^{t} x^{T}(\varphi) R x(\varphi) d \varphi=\zeta^{T}(t) \Pi_{i} \zeta(t),
\end{aligned}
$$

where $\Pi_{i}$ is defined in Theorem 6 and $\zeta(t)=\left[x^{T}(t), x^{T}(t-\right.$ $\left.\left.d_{i}(t)\right), x^{T}(t-\bar{d}), \int_{t-\bar{d}}^{t} x^{T}(\varphi) d \varphi, \omega^{T}(t)\right]^{T}$.

Noting that $\int_{t-d_{i}(t)}^{t} x(\varphi) Q x(\varphi) d \varphi \quad>\quad 0$ and $\int_{t-\bar{d}}^{t} x(\varphi) R x(\varphi) d \varphi>0$, it can be obtained that if $\Pi_{i}<0$ then $J<0$ holds, then

$$
\mathscr{L} V(t, i)-\alpha V(t, i)<\alpha \omega^{T}(t) \omega(t) .
$$

Pre- and postmultiplying (19) by $e^{-a t}$ yield

$$
\mathbb{E}\left\{e^{-a t} \mathscr{L} V(t, i)\right\}<a e^{-a t} \omega^{T}(t) \omega(t) .
$$

Moreover, by integrating (20) between 0 and $t$, this yields

$$
\mathbb{E}\left\{e^{-a t} \mathscr{L} V(t, i)\right\}<a e^{-a t} \omega^{T}(t) \omega(t)
$$

which means that

$$
\begin{aligned}
\mathbb{E}\{V(t, i)\}<e^{a t} \mathbb{E}\left\{V\left(0, r_{0}\right)\right\} \\
+a e^{a t} \int_{0}^{t} e^{-a \varphi} \omega^{T}(\varphi) \omega(\varphi) d \varphi .
\end{aligned}
$$

Denoting $\mathscr{P}_{i}=W^{-1 / 2} P_{i} W^{-1 / 2}, Q=W^{-1 / 2} Q W^{-1 / 2}$, and $\mathscr{R}=W^{-1 / 2} R W^{-1 / 2}$, it can be obtained that

$$
\begin{aligned}
& \mathbb{E}\left\{e^{a t} V\left(0, r_{0}\right)\right\}+a e^{a t} \int_{0}^{t} e^{-a \varphi} \omega^{T}(\varphi) \omega(\varphi) d \varphi \\
& =\mathbb{E}\left\{e^{a t} x^{T}(0) P_{i} x(0)\right. \\
& +e^{a t} \int_{-d_{i}(t)}^{0} \tilde{x}^{T}(\varphi) Q x(\varphi) d \varphi \\
& \left.+e^{a t} \int_{-\bar{d}}^{0} x^{T}(\varphi) R x(\varphi) d \varphi\right\} \\
& +a e^{a t} \int_{0}^{t} e^{-a \varphi} \omega^{T}(\varphi) \omega(\varphi) d \varphi \leq e^{a T} \lambda_{\max }\left(\mathscr{P}_{i}\right) \\
& +c_{1}+e^{a T} \bar{d} \lambda_{\max }(\mathscr{Q}) c_{1}+e^{a T} \bar{d} \lambda_{\max }(\mathscr{R}) c_{1} \\
& +\delta e^{a T}\left(1-e^{-a T}\right)=e^{a T}\left[c_{1}\left(\lambda_{1}+d \lambda_{2}+d \lambda_{3}\right)\right. \\
& \left.+\delta\left(1-e^{-a T}\right)\right] .
\end{aligned}
$$


On the other hand, the following condition holds:

$$
\begin{aligned}
& \mathbb{E}\{V(t, i)\}=\mathbb{E}\left\{x^{T}(t) P_{i} x(t)\right. \\
& +\int_{t-d_{i}(t)}^{t} x^{T}(\varphi) Q x(\varphi) d \varphi \\
& \left.+\int_{t-\bar{d}}^{t} x^{T}(\varphi) R x(\varphi) d \varphi\right\} \geq \mathbb{E}\left\{x^{T}(t) P_{i} x(t)\right\} \\
& \geq \lambda_{\min }\left(\mathscr{P}_{i}\right) \mathbb{E}\left\{x^{T}(t) W x(t)\right\} \\
& =\lambda_{4} \mathbb{E}\left\{x^{T}(t) W x(t)\right\} .
\end{aligned}
$$
that

Then, by using Dynkin's formula [22], it can be derived

$$
\begin{aligned}
\mathbb{E}\left\{x^{T}(t) W x(t)\right\} \\
\leq \frac{c_{1}\left(\lambda_{1}+d \lambda_{2}+d \lambda_{3}\right)+\delta\left(1-e^{-a T}\right)}{\lambda_{4} e^{-a T}}
\end{aligned}
$$

which means that system (5) is MSSFTB with respect to $\left(c_{1}, c_{2}, \delta, T, W\right)$ in the sense of Definition 2 and this completes the proof.

Remark 7. It should be noted that the positive matrix $W$ is used to address the state elements with different weights. Moreover, it can be observed that when $\omega(t) \equiv 0$, the concept of MSSFTB system will lead to MSSFTS system. The differences between the Lyapunov stability and MSSFTS system can be further shown from the above proof procedure.

Based on the obtained results in Theorem 6, the following theorem is presented to deal with the MSSFTP problem.

Theorem 8. For given positive constants $c_{1}, \delta$ and a symmetric matrix $W>0$, system (5) is MSSFTP with respect to $\left(c_{1}, c_{2}, \delta, T, W\right)$, if there exist a positive constant $c_{2}$ and modedependent symmetric matrices $P_{i}>0$, symmetric matrices $Q>0$ and $R>0$, such that $\bar{\Pi}_{i}<0$ holds for each mode $i \in \mathscr{I}$ and $c_{1}\left(\lambda_{1}+d \lambda_{2}+d \lambda_{3}\right)+\delta\left(1-e^{-a T}\right)<c_{2} \lambda_{4} e^{-a T}$, where

$$
\begin{aligned}
& \bar{\Pi}_{i} \triangleq\left[\begin{array}{cc}
\bar{\Pi}_{i 1} & \bar{\Pi}_{i 2} \\
* & \bar{\Pi}_{i 3}
\end{array}\right], \\
& \bar{\Pi}_{i 1} \triangleq \operatorname{sym}\left\{P_{i} A_{i}\right\}+\operatorname{sym}\left\{P_{i} B_{i} K_{i}\right\}+Q+R-\alpha P_{i} \\
& +\sum_{j=1}^{N} \pi_{i j} P_{j} \\
& \bar{\Pi}_{i 2} \triangleq\left[\begin{array}{cccc}
P_{i} A_{d i} & 0 & 0 & P_{i} B_{\omega i}-C_{i}^{T} \\
-(1-\mu) Q & 0 & 0 & -C_{d i}^{T}
\end{array}\right], \\
& \bar{\Pi}_{i 3} \triangleq \operatorname{diag}\left\{-R, \frac{\bar{\pi}}{\bar{d}} Q,-\beta I-\operatorname{sym}\left\{D_{\omega i}\right\}\right\} \text {, }
\end{aligned}
$$

with $\bar{\pi}, \lambda_{1}, \lambda_{2}, \lambda_{3}$, and $\lambda_{4}$ defined in Theorem 6 .
Proof. Choose the same Lyapunov-Krasovskii function candidate in proof of Theorem 6 and give the following function:

$$
\begin{aligned}
\bar{J} \triangleq & \mathscr{L} V(t, i) \\
& -\left[\alpha V(t, i)+\beta \omega^{T}(t) \omega(t)+2 y^{T}(t) \omega(t)\right] .
\end{aligned}
$$

Then, one has

$$
\begin{aligned}
& \bar{J}+\alpha \int_{t-d_{i}(t)}^{t} x(\varphi) \mathrm{Q} x(\varphi) d \varphi \\
& \quad+\alpha \int_{t-\bar{d}}^{t} x(\varphi) R x(\varphi) d \varphi=\zeta^{T}(t) \bar{\Pi}_{i} \zeta(t),
\end{aligned}
$$

where $\bar{\Pi}_{i}$ is defined in Theorem 8 and $\zeta(t)=\left[x^{T}(t), x^{T}(t-\right.$ $\left.\left.d_{i}(t)\right), x^{T}(t-\bar{d}), \int_{t-\bar{d}}^{t} x^{T}(\varphi) d \varphi, \omega^{T}(t)\right]^{T}$.

Noting that $\int_{t-d_{i}(t)}^{t} x(\varphi) Q x(\varphi) d \varphi \quad>\quad 0$ and $\int_{t-\bar{d}}^{t} x(\varphi) R x(\varphi) d \varphi>0$, it can be obtained that if $\bar{\Pi}_{i}<0$ then $\bar{J}<0$ holds, and then

$$
\mathscr{L} V(t, i)-\alpha V(t, i)<2 y^{T}(t) \omega(t)+\beta \omega^{T}(t) \omega(t) .
$$

Pre- and postmultiplying (29) by $e^{-a t}$ yield

$$
\begin{aligned}
\mathbb{E} & \left\{\mathscr{L}\left(e^{-a t} V(t, i)\right)\right\} \\
& <\mathbb{E}\left\{e^{-a t}\left[2 y^{T}(t) \omega(t)+\beta \omega^{T}(t) \omega(t)\right]\right\} .
\end{aligned}
$$

Consequently, under zero initial condition, the following inequality holds by integrating (30) between 0 and $T$ :

$$
\begin{array}{r}
\mathbb{E}\left\{e^{-a T} V(t, i)\right\}<\mathbb{E}\left\{2 \int_{0}^{T} e^{-a t} y^{T}(t) \omega(t) d t\right\} \\
+\beta \int_{0}^{T} e^{-a t} \omega^{T}(t) \omega(t) d t \\
<2 \mathbb{E}\left\{\int_{0}^{T} y^{T}(t) \omega(t) d t\right\} \\
+\beta e^{-a T} \int_{0}^{T} \omega^{T}(t) \omega(t) d t
\end{array}
$$

such that one has

$$
\begin{aligned}
0 & <\mathbb{E}\{V(t, i)\} \\
& <2 e^{a T} \mathbb{E}\left\{\int_{0}^{T} y^{T}(t) \omega(t) d t\right\}+\beta \int_{0}^{T} \omega^{T}(t) \omega(t) d t .
\end{aligned}
$$

Thus, it follows that

$$
2 \mathbb{E}\left\{\int_{0}^{T} y^{T}(t) \omega(t) d t\right\}>-\beta e^{-a T} \int_{0}^{T} \omega^{T}(t) \omega(t) d t .
$$

By letting $\gamma=\beta e^{-a T}$, the closed-loop system (4) can be MSSFTP with respect to $\left(c_{1}, c_{2}, \delta, T, W\right)$ according to Definition 3, which completes the proof. 
Remark 9. It should be pointed out that the relation between MSSFTB system and MSSFTP index is revealed by the proof of Theorem 8. Compared with the normal stability problem, the solutions derived from the theoretical results in the form of strict LMIs are more complex with MSSFTP index. Moreover, the mode-dependent time-varying delays are introduced such that the mathematical model can be more applicable. The obtained results show that the passivity theory can be effectively utilized to deal with the disturbance. Moreover, the minimum value of parameter $\gamma$ can be obtained by the convex optimization procedure in MATLAB.
In the following, the mode-dependent controller gain design procedure will be presented with standard matrix manipulation based on the results of Theorem 8 .

Theorem 10. For given positive constants $c_{1}, \delta$ and a symmetric matrix $W>0$, system (5) is MSSFTP with respect to $\left(c_{1}, c_{2}, \delta, T, W\right)$, if there exist positive constants $c_{2}, \kappa$ and symmetric matrices $P_{i}, Q>0$, and $R>0$ and matrices $\mathbb{K}_{i}$, such that $\widetilde{\Pi}_{i}<0$ holds for each mode $i \in \mathscr{I}$ and $c_{1}\left(1 / \kappa+4 d / \kappa^{2}\right)+$ $\delta\left(1-e^{-a T}\right)<c_{2} e^{-a T}, \kappa W^{-1}<\widetilde{P}_{i}<W^{-1}, 0<\widetilde{Q}<2 W^{-1}$, and $0<\widetilde{R}<2 W^{-1}$, where

$$
\begin{aligned}
& \widetilde{\Pi}_{i} \triangleq\left[\begin{array}{cc}
\widetilde{\Pi}_{i 1} & \widetilde{\Pi}_{i 2} \\
* & \widetilde{\Pi}_{i 3}
\end{array}\right] \\
& \widetilde{\Pi}_{i 1} \triangleq\left[\begin{array}{cc}
\operatorname{sym}\left\{A_{i} \widetilde{P}_{i}\right\}+\operatorname{sym}\left\{B_{i} \mathbb{K}_{i}\right\}+\widetilde{Q}+\widetilde{R}-\alpha \widetilde{P}_{i}+\pi_{i i} \widetilde{P}_{i} & A_{d i} \widetilde{P}_{i} \\
* & -(1-\mu) \widetilde{Q}
\end{array}\right], \\
& \widetilde{\Pi}_{i 2} \triangleq\left[\begin{array}{cccccc}
0 & 0 & B_{\omega i}-\widetilde{P}_{i} C_{i}^{T} & \sqrt{\pi_{i 1}} \widetilde{P}_{i} & \cdots & \sqrt{\pi_{i N}} \widetilde{P}_{i} \\
0 & 0 & -\widetilde{P}_{i} C_{d i}^{T} & 0 & \cdots & 0
\end{array}\right] \text {, } \\
& \widetilde{\Pi}_{i 3} \triangleq \operatorname{diag}\left\{-\widetilde{R}, \frac{\bar{\pi}}{\overline{\bar{d}}} \widetilde{Q},-\beta I-\operatorname{sym}\left\{D_{\omega i}\right\},-\widetilde{P}_{1}, \ldots,-\widetilde{P}_{N}\right\} \text {, }
\end{aligned}
$$

with $\bar{\pi}, \lambda_{1}, \lambda_{2}, \lambda_{3}$, and $\lambda_{4}$ defined in Theorem 6 .

Moreover, the desired mode-dependent passification controller gains can be obtained by $K_{i}=\mathbb{K}_{i} \widetilde{P}_{i}^{-1}$.

$$
\begin{aligned}
& \widehat{\Pi}_{i} \triangleq\left[\begin{array}{cc}
\widehat{\Pi}_{i 1} & \widehat{\Pi}_{i 2} \\
* & \widehat{\Pi}_{i 3}
\end{array}\right], \\
& \widehat{\Pi}_{i 1} \triangleq\left[\begin{array}{ccc}
\operatorname{sym}\left\{P_{i} A_{i}\right\}+\operatorname{sym}\left\{P_{i} B_{i} K_{i}\right\}+Q+R-\alpha P_{i}+\pi_{i i} P_{i} & P_{\mathrm{i}} A_{d i} & 0 \\
* & -(1-\mu) Q & 0 \\
* & * & -R
\end{array}\right] \text {, } \\
& \widehat{\Pi}_{i 2} \triangleq\left[\begin{array}{ccccc}
0 & P_{i} B_{\omega i}-C_{i}^{T} & \sqrt{\pi_{i 1}} I & \cdots & \sqrt{\pi_{i N}} I \\
0 & -C_{d i}^{T} & 0 & \cdots & 0 \\
0 & 0 & 0 & \cdots & 0
\end{array}\right] \\
& \widehat{\Pi}_{i 3} \triangleq\left[\begin{array}{ccccc}
\overline{\bar{\pi}} & 0 & 0 & 0 & 0 \\
* & -\beta I-\operatorname{sym}\left\{D_{\omega i}\right\} & 0 & 0 & 0 \\
* & * & -P_{1}^{-1} & 0 & 0 \\
* & * & * & \ddots & 0 \\
* & * & * & * & -P_{N}^{-1}
\end{array}\right] .
\end{aligned}
$$

Pre- and postmultiply $\widehat{\Pi}_{i}<0$ by $\operatorname{diag}\left\{P_{i}^{-1}, P_{i}^{-1}, P_{i}^{-1}, P_{i}^{-1}\right.$, $I, \ldots, I\}$ and let $\widetilde{P}_{i}=P_{i}^{-1}, \widetilde{Q}=P_{i}^{-1} Q P_{i}^{-1}$, and $\widetilde{R}$ and
$K_{i} \widetilde{P}_{i}^{-1}=\mathbb{K}_{i}$. Furthermore, it follows that $\lambda_{\max }\left(\widetilde{P}_{i}\right)<1 / \kappa$ and $\lambda_{\min }\left(\widetilde{P}_{i}\right)>1$. Moreover, one has $\lambda_{\max }(\widetilde{Q})<\left(2 / \kappa^{2}\right)$ 
and $\lambda_{\max }(\widetilde{R})<\left(2 / \kappa^{2}\right)$. The rest of the proof is similar to the proof of Theorem 8 . Then, the desired mode-dependent passification controller gains can be directly obtained, which completes the proof.

\section{Numerical Examples}

In this section, two numerical examples are presented to verify our designed mode-dependent passification controller.

Example 1. Consider the Markovian jumping system in the form of (1) with two modes. For mode 1, the dynamics of the system are described as

$$
\begin{aligned}
A_{1} & =\left[\begin{array}{cc}
1.5 & 1 \\
1 & -2
\end{array}\right], \\
A_{d 1} & =\left[\begin{array}{cc}
1 & 0.1 \\
-1 & 1
\end{array}\right], \\
B_{1} & =\left[\begin{array}{c}
1 \\
0.9
\end{array}\right], \\
B_{\omega 1} & =\left[\begin{array}{l}
1 \\
1
\end{array}\right], \\
C_{1} & =\left[\begin{array}{ll}
0.8 & 0.5
\end{array}\right], \\
C_{d 1} & =\left[\begin{array}{ll}
1.2 & 1.3
\end{array}\right], \\
D_{\omega 1} & =0.15,
\end{aligned}
$$

and the time-varying delay satisfies $d_{1}(t)=0.2+0.2 \sin t$.

For mode 2, the dynamics of the system are described as

$$
\begin{aligned}
A_{2} & =\left[\begin{array}{ll}
1 & 0.5 \\
1 & -1
\end{array}\right], \\
A_{d 2} & =\left[\begin{array}{ll}
0.4 & -0.2 \\
0.3 & 0.8
\end{array}\right], \\
B_{2} & =\left[\begin{array}{l}
1.2 \\
0.8
\end{array}\right], \\
B_{\omega 2} & =\left[\begin{array}{l}
0.8 \\
1.2
\end{array}\right], \\
C_{2} & =\left[\begin{array}{ll}
1 & 0.7
\end{array}\right], \\
C_{d 2} & =\left[\begin{array}{ll}
1.1 & 1.2
\end{array}\right], \\
D_{\omega 2} & =0.2,
\end{aligned}
$$

and the time-varying delay satisfies $d_{2}(t)=0.3+0.1$ cost . Then, it can be obtained that $\bar{d}=0.5$ and $\mu=0.2$.

Moreover, the transition probability matrix is given as

$$
\Theta=\left[\begin{array}{cc}
-0.6 & 0.6 \\
0.4 & -0.4
\end{array}\right] \text {. }
$$

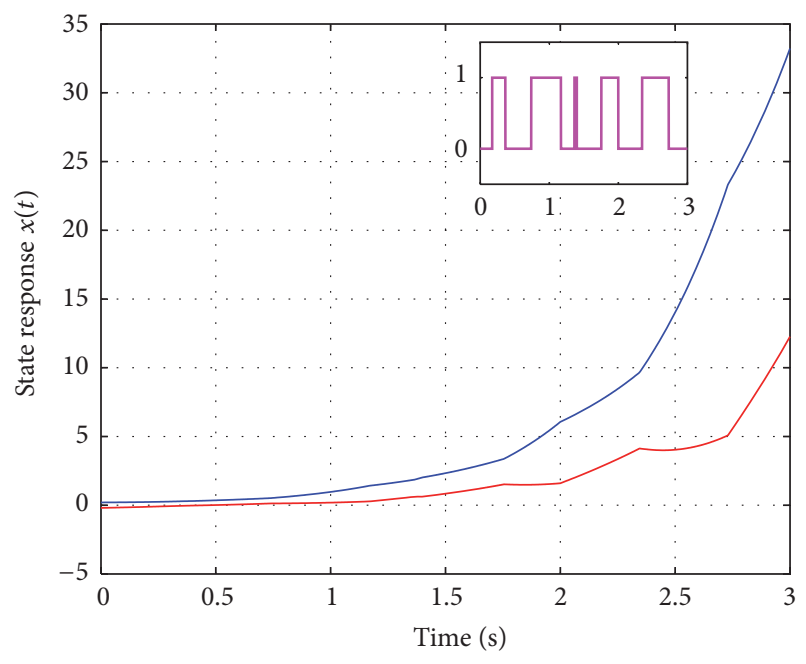

FIgURE 1: State response of the open-loop system.

The scalars $\left(c_{1}, c_{2}, \delta, T, W\right)$ are given as $(0.15,5.5$, $0.4,3 s, I)$. The scalars $\alpha$ and $\beta$ are chosen as $\alpha=0.4$ and $\beta=1$, respectively. Then, one has the MSSFTP performance index $\gamma$ is calculated by 0.3012 . The disturbance input $\omega(t)$ is assumed to be $0.2 \sin t$.

With the above parameters, the feasible solutions can be found by solving the LMIs in Theorem 10 and the desired mode-dependent controller gains can be obtained as

$$
\begin{aligned}
& K_{1}=\left[\begin{array}{ll}
-17.2468 & -10.0573
\end{array}\right], \\
& K_{2}=\left[\begin{array}{ll}
-13.9580 & -8.1791
\end{array}\right] .
\end{aligned}
$$

In order to demonstrate the validity of the obtained results, the designed controller is applied to the above system. Given initial states as $[0.2,-0.2]^{T}$, Figures 1 and 2 show the simulation results of the corresponding state trajectories of the open-loop and closed-loop system dynamics with transition probability modes, respectively.

Figures 3 and 4 illustrate the corresponding state finitetime evolution curves, respectively.

Furthermore, Figure 5 depicts the effect on $y(t)$ from the disturbance $\omega(t)$ within $[0,3 s]$. It can be calculated that $2 \int_{0}^{3} y^{T}(t) \omega(t) d t=0.0028$ and $\int_{0}^{3} \omega^{T}(t) \omega(t) d t=0.628$, such that

$$
\frac{2 \int_{0}^{3} y^{T}(t) \omega(t) d t}{\int_{0}^{3} \omega^{T}(t) \omega(t) d t} \geq-\gamma
$$

which implies that the developed mode-dependent passification controller can achieve the MSSFTP index and supports our obtained theoretical results. 


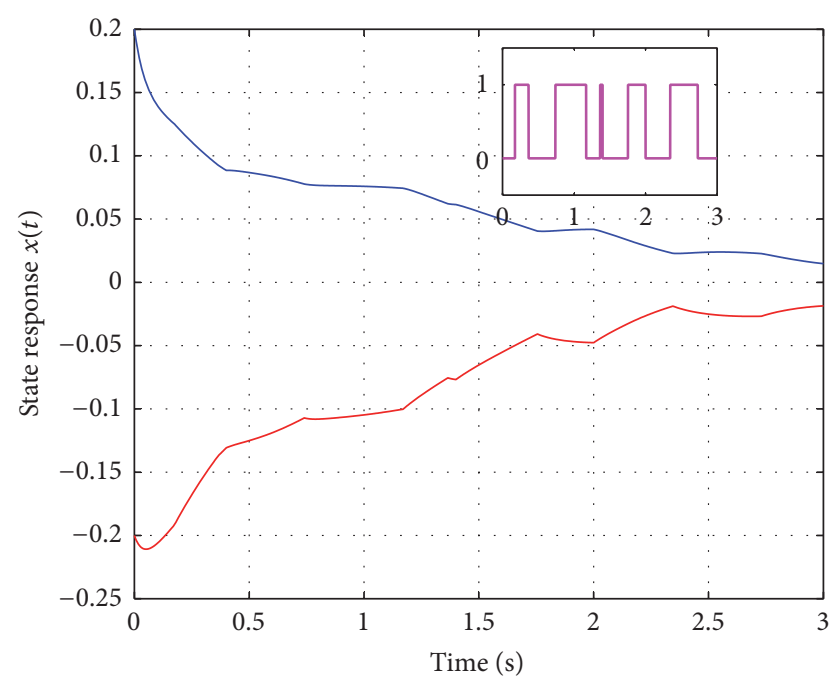

FIGURE 2: State response of the closed-loop system.

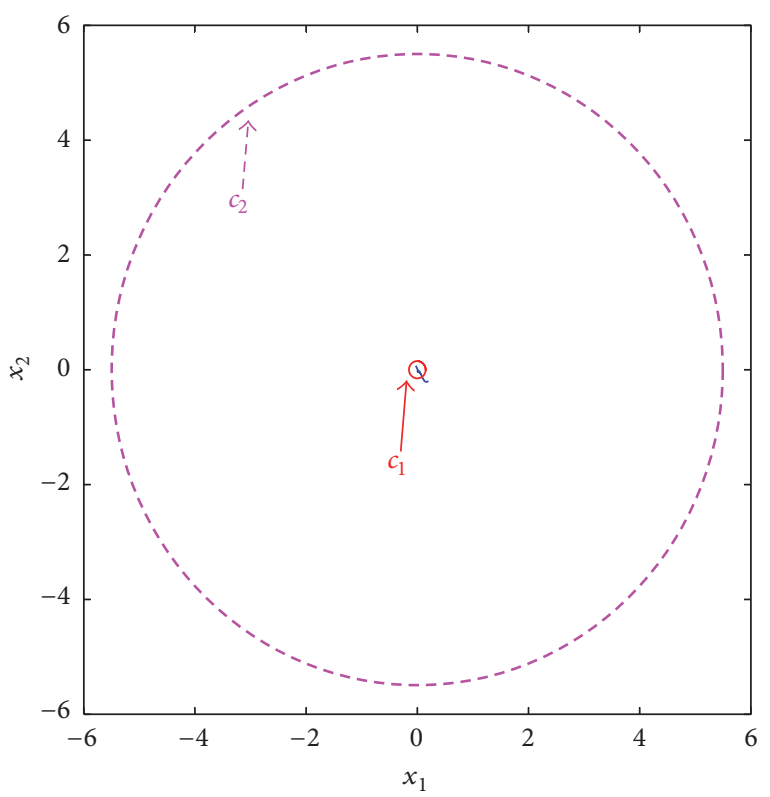

Figure 3: Curves of $x^{T}(t) W x(t)$.

Example 2. Another practical example of VTOL (vertical take-off and landing) helicopter is provided. The system dynamics can be modeled by $[23,24]$

$$
\begin{aligned}
& \dot{x}(t)=A(r(t)) x(t)+B(r(t)) u(t)+B_{\omega}(r(t)) \omega(t), \\
& y(t)=C(r(t)) x(t)+D_{\omega}(r(t)) \omega(t),
\end{aligned}
$$

where the state vector $x(t)=\left[x_{1}(t), x_{2}(t), x_{3}(t), x_{4}(t)\right]^{T} \in$ $\mathbb{R}^{4}, x_{1}(t)$ denotes the horizontal velocity, $x_{2}(t)$ denotes the vertical velocity, $x_{3}(t)$ denotes the pitch rate, and $x_{4}(t)$ denotes the pitch angle, $u(t)$ represents the control input,

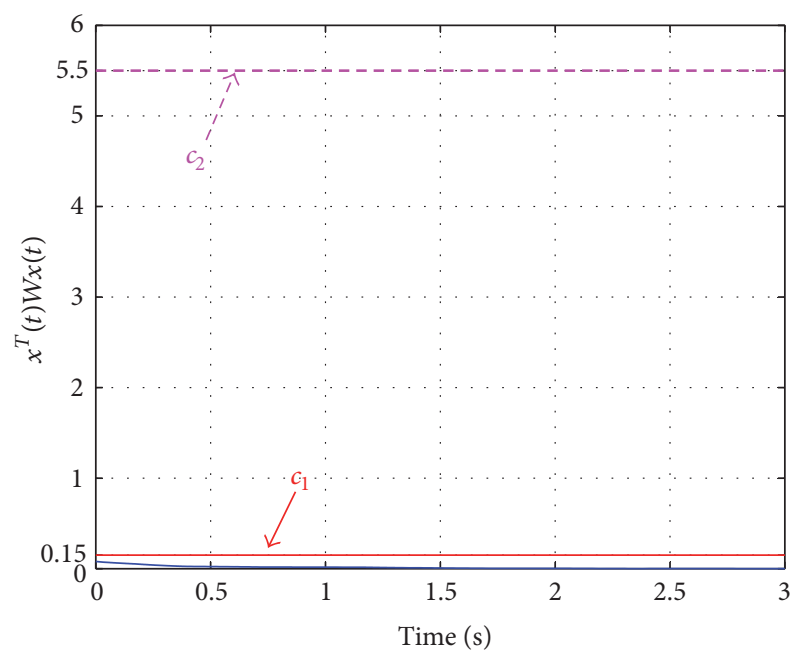

Figure 4: Time history of $x^{T}(t) W x(t)$.

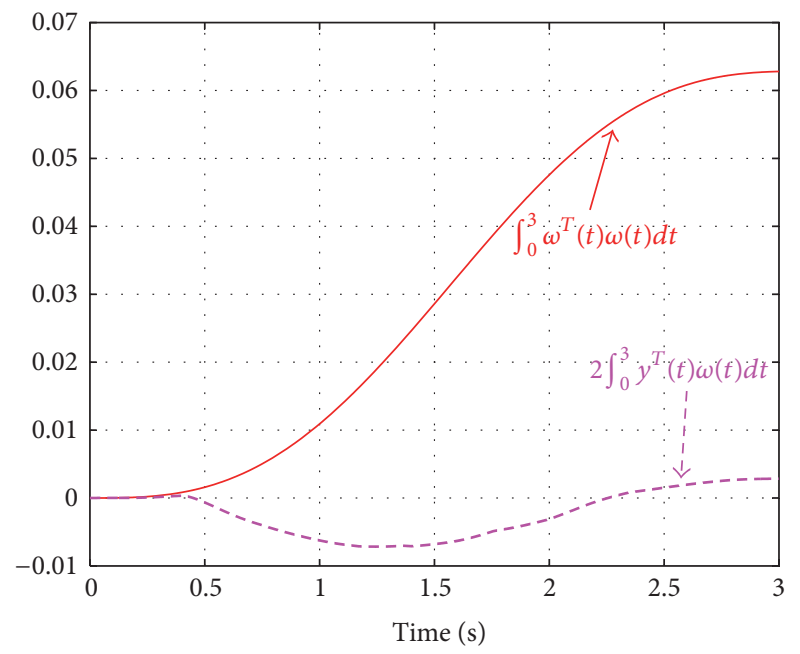

FIgure 5: Time history of $2 \int_{0}^{3} y^{T}(t) \omega(t) d t$ and $\int_{0}^{3} \omega^{T}(t) \omega(t) d t$.

and $\omega(t)$ represents the external disturbance and the system matrices are given as

$$
\begin{aligned}
& A_{1}=\left[\begin{array}{cccc}
-0.03660 & 0.0271 & 0.0188 & -0.4555 \\
0.0482 & -1.01 & 0.0024 & -4.0208 \\
0.1002 & 0.3681 & -0.707 & 1.4200 \\
0 & 0 & 1 & 0
\end{array}\right], \\
& A_{2}=\left[\begin{array}{cccc}
-0.03660 & 0.0271 & 0.0188 & -0.4555 \\
0.0482 & -1.01 & 0.0024 & -4.0208 \\
0.1002 & 0.0664 & -0.707 & 0.1198 \\
0 & 0 & 1 & 0
\end{array}\right],
\end{aligned}
$$




$$
\begin{aligned}
A_{3} & =\left[\begin{array}{cccc}
-0.03660 & 0.0271 & 0.0188 & -0.4555 \\
0.0482 & -1.01 & 0.0024 & -4.0208 \\
0.1002 & 0.5047 & -0.707 & 2.5460 \\
0 & 0 & 1 & 0
\end{array}\right], \\
B_{1} & =\left[\begin{array}{c}
0.4422 \\
3.5446 \\
-5.5200 \\
0
\end{array}\right], \\
B_{2} & =\left[\begin{array}{c}
0.4422 \\
0.9775 \\
-5.5200 \\
0
\end{array}\right], \\
B_{3} & =\left[\begin{array}{c}
0.4422 \\
5.1120 \\
-5.5200 \\
0
\end{array}\right], \\
C_{1} & =C_{2}=C_{3}=\left[\begin{array}{ll}
0 & 0.5 \\
D_{\omega 1} & =D_{\omega 2}=D_{\omega 3}=0.3 .
\end{array}\right], \\
B_{\omega 1} & =B_{\omega 2}=B_{\omega 3}=\left[\begin{array}{c}
0.1761 \\
-7.5922 \\
4.4900 \\
0
\end{array}\right],
\end{aligned}
$$

For simplicity, let $r(t)$ denote a continuous-time Markov process with three modes which corresponds to the airspeeds 135,60 , and 170 knots. The transition probability matrix is given by

$$
\Theta=\left[\begin{array}{ccc}
-0.9 & 0.3 & 0.6 \\
0.7 & -1.2 & 0.5 \\
0.8 & 0.5 & -1.3
\end{array}\right] \text {. }
$$

The scalars $\left(c_{1}, c_{2}, \delta, T, W\right)$ are given as $(50,5000,4,5 s, I)$. The scalars $\alpha$ and $\beta$ are chosen as $\alpha=0.1$ and $\beta=60$, respectively. The MSSFTP performance index $\gamma$ can be calculated by 36.3918 . The initial states are set as $[4,5,2,2]^{T}$. The disturbance input $\omega(t)$ is assumed to be $0.5 \sin t$. Similarly, the feasible solutions can be found by solving the LMIs in Theorem 10 and the desired mode-dependent controller gains can be obtained as

$$
\begin{aligned}
& K_{1}=\left[\begin{array}{llll}
-22.8723 & -95.6256 & 141.0474 & 124.0033
\end{array}\right], \\
& K_{2}=\left[\begin{array}{llll}
-21.9497 & -68.3356 & 178.5414 & 191.1515
\end{array}\right], \\
& K_{3}=\left[\begin{array}{llll}
-20.8118 & -91.3390 & 136.9706 & 113.7022
\end{array}\right] .
\end{aligned}
$$

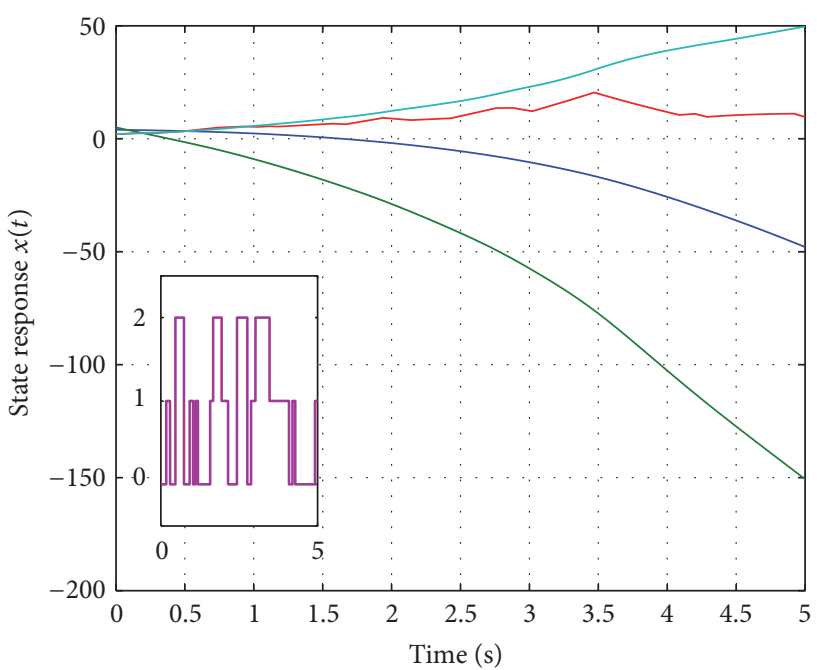

FIGURE 6: State response of the open-loop VTOL helicopter system.

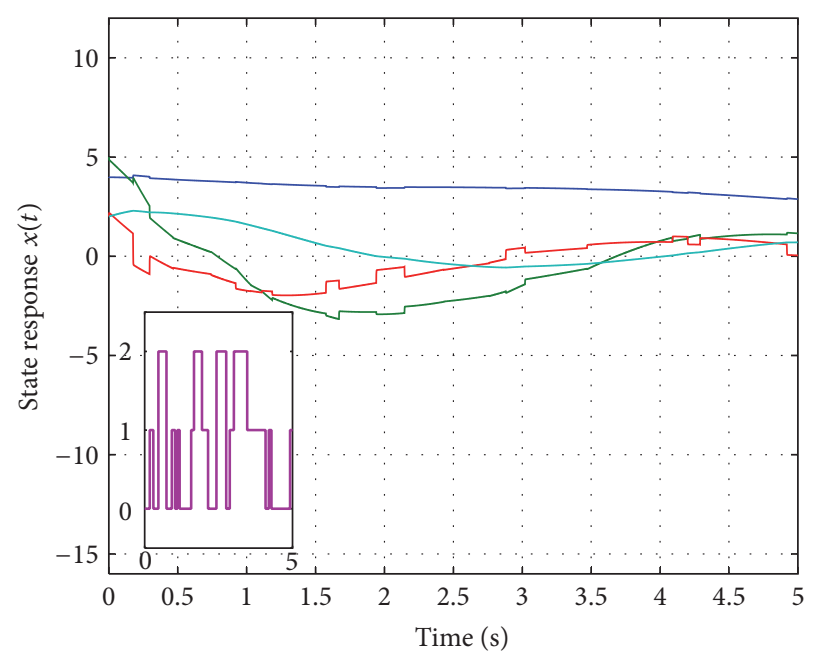

FIGURE 7: State response of the closed-loop VTOL helicopter system.

Moreover, it can be obtained that $2 \int_{0}^{5} y^{T}(t) \omega(t) d t=-1.217$ and $\int_{0}^{5} \omega^{T}(t) \omega(t) d t=0.659$, such that

$$
\frac{2 \int_{0}^{5} y^{T}(t) \omega(t) d t}{\int_{0}^{5} \omega^{T}(t) \omega(t) d t} \geq-\gamma
$$

Correspondingly, it can be seen from Figures 6-9 that the designed finite-time passification controller can achieve the prescribed passivity performance of the resulting closed-loop VTOL helicopter system.

\section{Conclusion}

In this paper, the finite-time passivity and passification problem of Markovian jumping systems with mode-dependent 


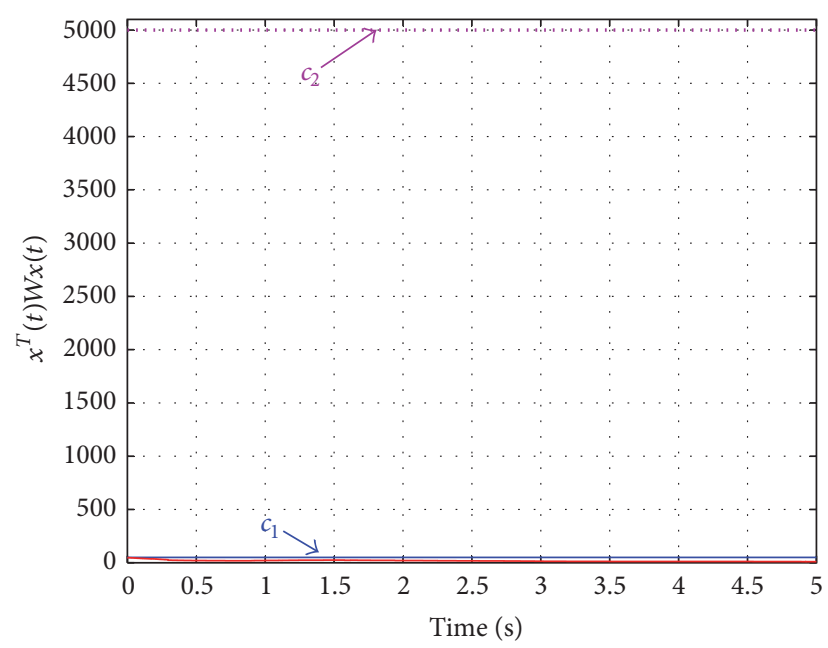

FIGURE 8: Time history of $x^{T}(t) W x(t)$.

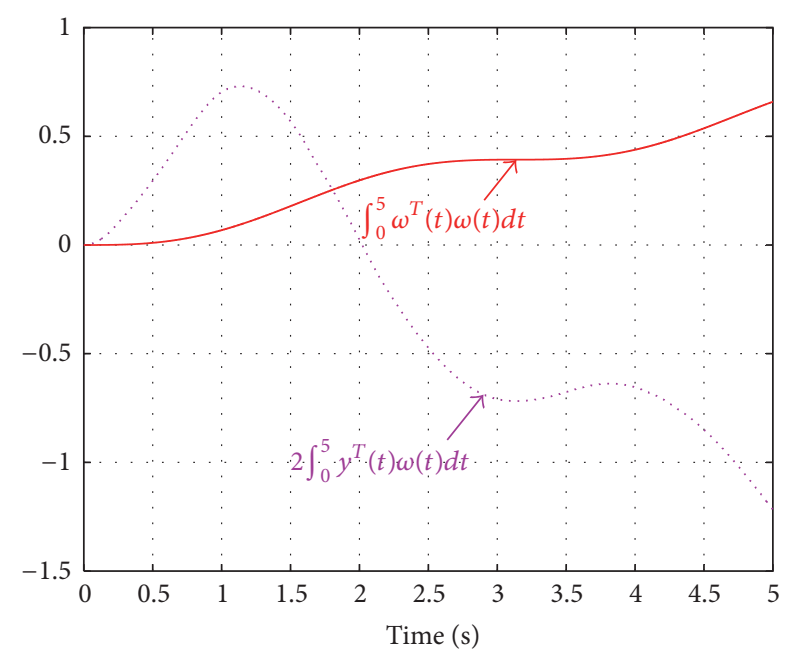

Figure 9: Time history of $2 \int_{0}^{5} y^{T}(t) \omega(t) d t$ and $\int_{0}^{5} \omega^{T}(t) \omega(t) d t$.

time-varying delays is considered. By applying the LyapunovKrasovskii functional method, delay-dependent passivity conditions are established to guarantee the MSSFTP index with respect to $\left(c_{1}, c_{2}, \delta, T, W\right)$. For the finite-time passification problem, mode-dependent controller is designed to achieve the prescribed passivity performance of the resulting closed-loop Markovian jumping system. In the end, two numerical examples are presented to illustrate the effectiveness of our developed findings. Our interesting further research direction includes extending our current obtained results to the cases with deficient mode transition.

\section{Conflicts of Interest}

The author declares that there is no conflict of interests regarding the publication of this paper.

\section{Acknowledgments}

This work was supported by the Fundamental Research Funds for the Central Universities under Grant FRF-TP-15$115 \mathrm{~A} 1$.

\section{References}

[1] Q. Zhu and J. Cao, "Stochastic stability of neural networks with both Markovian jump parameters and continuously distributed delays," Discrete Dynamics in Nature and Society, vol. 2009, Article ID 490515, 20 pages, 2009.

[2] T. Sathyan and T. Kirubarajan, "Markov-jump-system-based secure chaotic communication," IEEE Transactions on Circuits and Systems. I. Regular Papers, vol. 53, no. 7, pp. 1597-1609, 2006.

[3] H. R. Karimi, "A sliding mode approach to $H_{\infty}$ synchronization of master-slave time-delay systems with Markovian jumping parameters and nonlinear uncertainties," Journal of the Franklin Institute. Engineering and Applied Mathematics, vol. 349, no. 4, pp. 1480-1496, 2012.

[4] L. Zhang, E.-K. Boukas, and J. Lam, "Analysis and synthesis of Markov jump linear systems with time-varying delays and partially known transition probabilities," Institute of Electrical and Electronics Engineers. Transactions on Automatic Control, vol. 53, no. 10, pp. 2458-2464, 2008.

[5] F. Li, L. Wu, and P. Shi, "Stochastic stability of semi-Markovian jump systems with mode-dependent delays," International Journal of Robust and Nonlinear Control, vol. 24, no. 18, pp. 33173330, 2014.

[6] Y. Wang, P. Shi, Q. Wang, and D. Duan, "Exponential Hfiltering for singular markovian jump systems with mixed modedependent time-varying delay," IEEE Transactions on Circuits and Systems I: Regular Papers, vol. 60, no. 9, pp. 2440-2452, 2013.

[7] T. H. Lee, S. Lakshmanan, J. H. Park, and P. Balasubramaniam, "State estimation for genetic regulatory networks with modedependent leakage delays, time-varying delays, and markovian jumping parameters,' IEEE Transactions on Nanobioscience, vol. 12, no. 4, pp. 363-375, 2013.

[8] H. Shen, S. Xu, J. Lu, and J. Zhou, "Passivity-based control for uncertain stochastic jumping systems with mode-dependent round-trip time delays," Journal of the Franklin Institute, vol. 349, no. 5, pp. 1665-1680, 2012.

[9] G. Nagamani and S. Ramasamy, "Stochastic dissipativity and passivity analysis for discrete-time neural networks with probabilistic time-varying delays in the leakage term," Applied Mathematics and Computation, vol. 289, pp. 237-257, 2016.

[10] H. Gao, T. Chen, and T. Chai, "Passivity and passification for networked control systems," SIAM Journal on Control and Optimization, vol. 46, no. 4, pp. 1299-1322, 2007.

[11] C. Li, H. Zhang, and X. Liao, "Passivity and passification of fuzzy systems with time delays," Computers and Mathematics with Applications, vol. 52, no. 6-7, pp. 1067-1078, 2006.

[12] J. Liang, Z. Wang, and X. Liu, "On passivity and passification of stochastic fuzzy systems with delays: the discrete-time case," IEEE Transactions on Systems, Man, and Cybernetics, Part B: Cybernetics, vol. 40, no. 3, pp. 964-969, 2010.

[13] B. Jiang, C. Gao, and J. Xie, "Passivity based sliding mode control of uncertain singular Markovian jump systems with time-varying delay and nonlinear perturbations," Applied Mathematics and Computation, vol. 271, pp. 187-200, 2015.

[14] Z.-G. Wu, J. H. Park, H. Su, and J. Chu, "Passivity analysis of Markov jump neural networks with mixed time-delays and 
piecewise-constant transition rates," Nonlinear Analysis. Real World Applications. An International Multidisciplinary Journal, vol. 13, no. 5, pp. 2423-2431, 2012.

[15] Z.-G. Wu, P. Shi, H. Su, and J. Chu, "Passivity analysis for discrete-time stochastic markovian jump neural networks with mixed time delays," IEEE Transactions on Neural Networks, vol. 22, no. 10, pp. 1566-1575, 2011.

[16] B. Zhang, W. X. Zheng, and S. Xu, "Delay-dependent passivity and passification for uncertain Markovian jump systems with time-varying delays," International Journal of Robust and Nonlinear Control, vol. 22, no. 16, pp. 1837-1852, 2012.

[17] Z. Xiang, Y.-N. Sun, and M. S. Mahmoud, "Robust finitetime Ho control for a class of uncertain switched neutral systems," Communications in Nonlinear Science and Numerical Simulation, vol. 17, no. 4, pp. 1766-1778, 2012.

[18] N. Jiang, X. Liu, W. Yu, and J. Shen, "Finite-time stochastic synchronization of genetic regulatory networks," Neurocomputing, vol. 167, pp. 314-321, 2015.

[19] H. Liu and G. Zhou, "Finite-time sampled-data control for switching T-S fuzzy systems," Neurocomputing, vol. 166, pp. 294-300, 2015.

[20] J. Cheng, H. Zhu, S. Zhong, F. Zheng, and Y. Zeng, "Finite-time filtering for switched linear systems with a mode-dependent average dwell time," Nonlinear Analysis. Hybrid Systems, vol. 15, pp. 145-156, 2015.

[21] K. Gu, J. Chen, and V. L. Kharitonov, Stability of Time-Delay Systems, Springer Science \& Business Media, Berlin, Germany, 2003.

[22] S. Ma, C. Zhang, and S. Zhu, "Robust stability for discretetime uncertain singular Markov jump systems with actuator saturation," IET Control Theory \& Applications, vol. 5, no. 2, pp. 255-262, 2011.

[23] J. Qiu, Y. Wei, and H. R. Karimi, "New approach to delaydependent $\mathrm{H}_{\infty}$ control for continuous-time Markovian jump systems with time-varying delay and deficient transition descriptions," Journal of the Franklin Institute. Engineering and Applied Mathematics, vol. 352, no. 1, pp. 189-215, 2015.

[24] L. Rong, X. Peng, and B. Zhang, "A reduced-order fault detection filtering approach for continuous-time markovian jump systems with polytopic uncertainties," Complexity, vol. 2017, Article ID 4927453, 14 pages, 2017. 


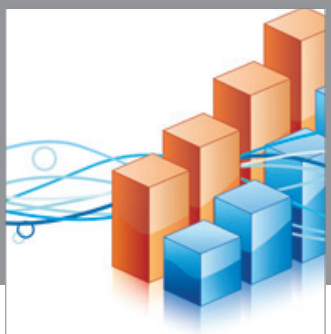

Advances in

Operations Research

vatem alat4

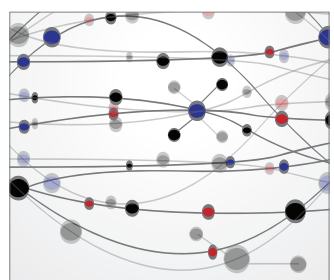

\section{The Scientific} World Journal
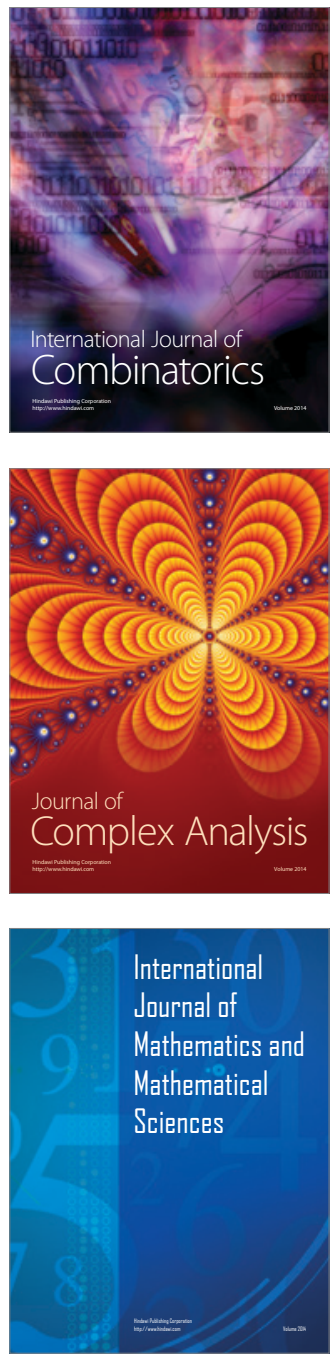
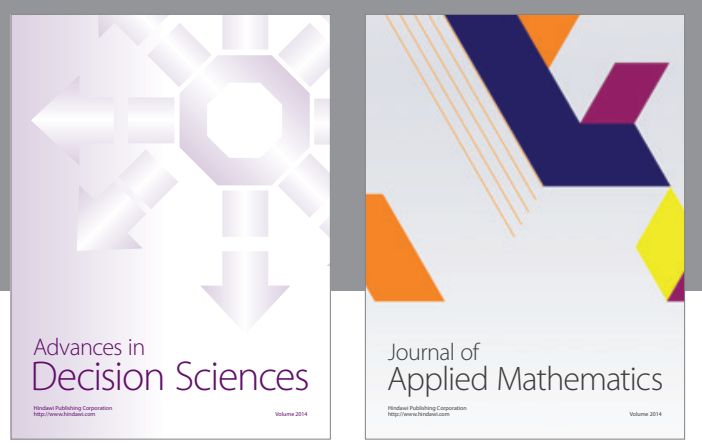

Algebra

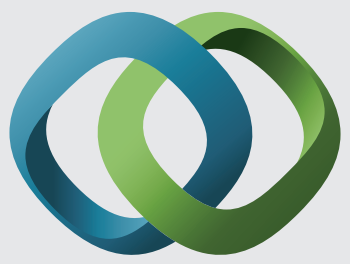

\section{Hindawi}

Submit your manuscripts at

https://www.hindawi.com
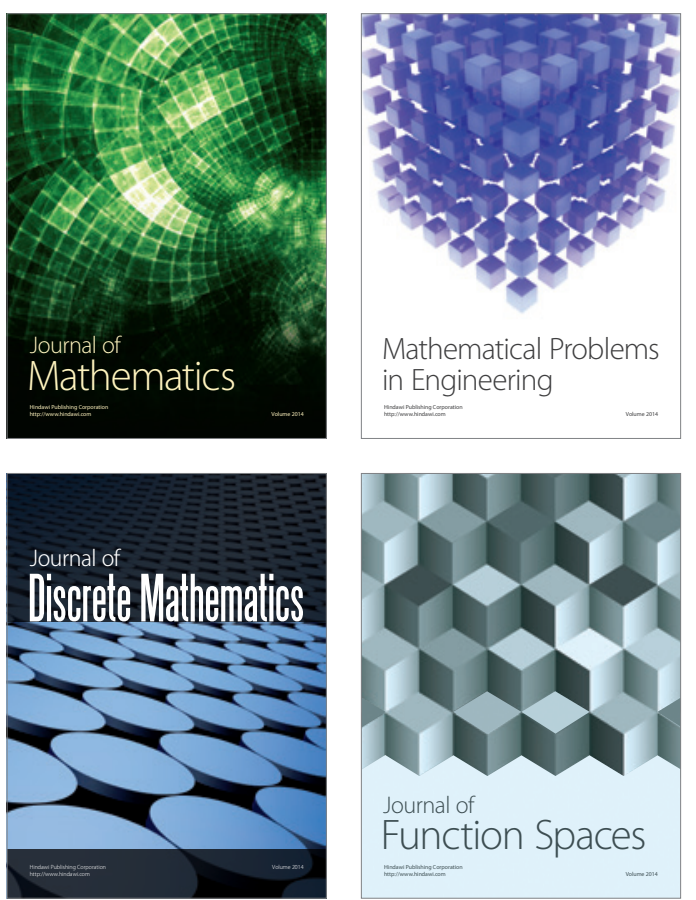

Mathematical Problems in Engineering
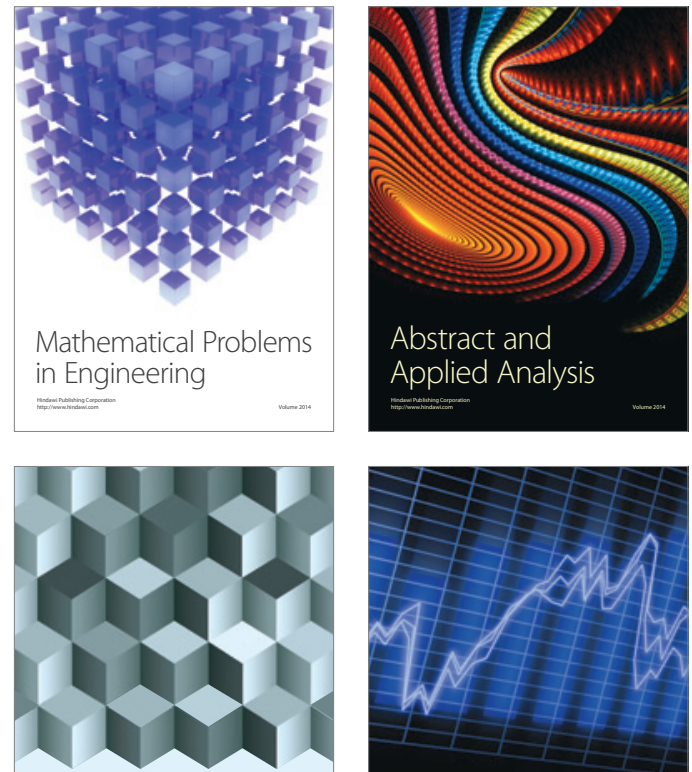

Journal of

Function Spaces

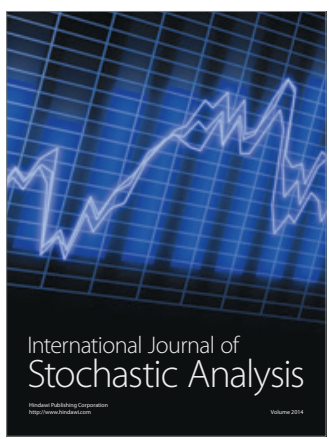

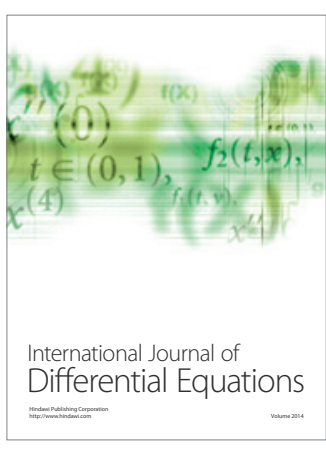
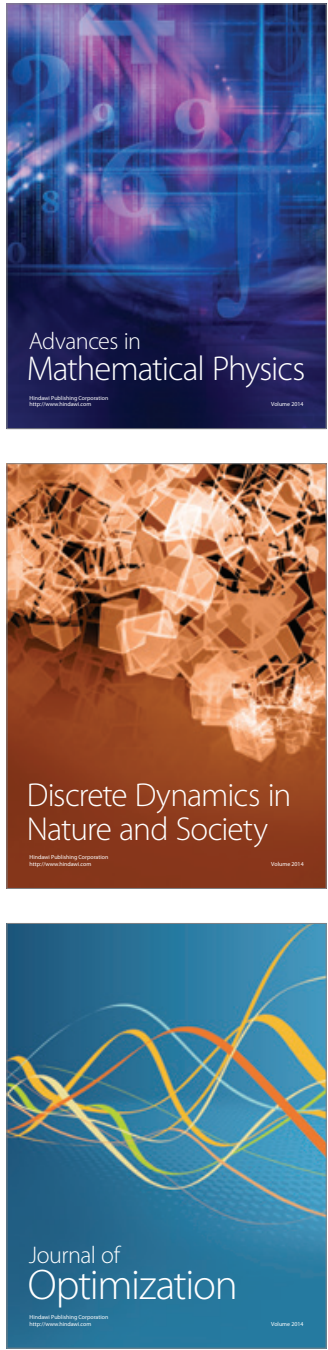